\title{
Factores de Riesgo Ergonómico en Personal de Atención Hospitalaria en Chile
}

\author{
ERGONOMIC RISK FACTORS IN HOSPITAL CARE IN CHILE
}

\author{
Valeria Paz Bravo Carrasco', Jorge Rodrigo Espinoza Bustos ${ }^{1}$ \\ 1. Departamento: Facultad de Ciencias Biológicas, Unidad de Ergonomía, Universidad de Concepción.
}

\section{RESUMEN}

Los factores de riesgo ergonómico en el personal de atención hospitalaria en Chile corresponden a una problemática actual en donde se identifican factores de riesgo de índole física y mental, generando falencias en el estado de salud de los trabajadores. Por esto, se identifican elementos en el lugar de trabajo como deficiencias biológicas, físicas y químicas, factores de riesgo psicosociales y organizacionales en el proceso de atención en salud.

Esta revisión busca identificar riesgos en actividades hospitalarias, definiciones de conceptos pertinentes al tema, identificar estudios que evalúen riesgos ocupacionales en el puesto de trabajo, dolencias músculo-esqueléticas, sistema de trabajo y la carga mental asociada. Por esto, se analiza la séptima encuesta laboral 2011 obteniendo un 18,6\% en organizaciones con trabajadores expuestos a posturas incómodas, $14,2 \%$ en movimientos repetitivos, $23,7 \%$ con exposición a turnos y un 10,7\% con exposición a ruído en el ambiente físico.

Posteriormente, se analiza una comparación del sistema de turnos entre el sector público y privado, encuestando a una muestra de 44 trabajadores de un hospital privado y 43 trabajadores de un hospital público, concluyendo que no se presenta riesgo significativo en ambas. Respecto a la carga mental, se analiza una investigación de 782 trabajadores, concluyendo la existencia de niveles de estrés psicosocial en los trabajadores de la salud.

En conclusión, es importante desarrollar investigación y realizar un seguimiento anual sobre el estado de salud de la población chilena perteneciente al área hospitalaria, con el objetivo de identificar variables deficientes y mejorar las condiciones del personal.

(Bravo V, Espinoza J, 2016. Factores de Riesgo Ergonómico en Personal de Atención Hospitalaria en Chile. Cienc Trab. Sep-Dic; 18 [57]: 150-153).

Palabras claves: SALUD, PERSONAL, ESTRÉS, TRASTORNOS MÚSCULO-ESQUELÉTICOS.

\section{ABSTRACT}

The ergonomic risk factors in hospital care personnel in Chile correspond to a current problem in which physical and mental risk factors are identified, gene rating shortcomings in the health status of workers. For this reason, elements in the workplace are identified as biological, physical and chemical deficiencies, psychosocial and organizational risk factors in the health care process.

This review seeks to identify risks in hospital activities, definitions of concepts pertinent to the subject, to identify studies that evaluate occupational hazards in the workplace, musculoskeletal conditions, work system and the associated mental load. For this reason, the seventh labor survey 2011 is analyzed, obtaining $18.6 \%$ in organizations with workers exposed to uncomfortable postures, $14.2 \%$ in repetitive movements, $23.7 \%$ with shift exposure and 10.7\% with exposure to Noise in the physical environment.

Subsequently, a comparison of the shift system between the public and private sector is analyzed by surveying a sample of 44 employees of a private hospital and 43 workers of a public hospital, concluding that there is no significant risk in both. With respect to the mental load, an investigation of 782 workers is analyzed concluding the existence of levels of psychosocial stress in the health workers.

In conclusion, it is important to develop research and annual monitoring of the health status of the Chilean population in the hospital area, in order to identify poor variables and improve staff conditions.

Key words: HEALTH, PERSONAL, STRESS, MUSCULOSKELETAL DISORDERS

Correspondencia / Correspondence:

Valeria Paz Bravo Carrasco

3 oriente 6 norte, edificio alameda \# 1015, depto. 502 Talca, Chile.

e-mail: vbravocarrasco@gmail.com

Jorge Rodrigo Espinoza Bustos

Campos de Bellavista $n^{\circ} 402$ Torre 10 depto. 303,

Condominio Los Cipreses 5. Concepción, Chile.

Tel.: +56-41-2204426

e-Mail: jespinozab@udec.cl

Recibido: 13 de septiembre de 2016 / Aceptado: 24 de noviembre de 2016

\section{INTRODUCCIÓN}

Los factores de riesgo ergonómico en el personal de atención hospitalaria en Chile se manifiestan como una problemática actual que se adquiere en algunos centros del país, en donde la demanda de la jornada laboral es elevada y el trabajador va desvalorizando su salud, trayendo como consecuencia repercusiones a nivel físico y psicológico, en primera instancia.

$\mathrm{Al}$ abordar esta temática, se demuestra evidencia en estudios desarrollados sobre el nivel de trabajo, problemas y dificultades que acarrea, lo que la convierte en un área de investigación interesante, identificando los aspectos mayoritarios para connotar en esta revisión.

El objetivo es lograr principalmente un mayor conocimiento sobre 
los riesgos ergonómicos de la atención hospitalaria a través de investigaciones efectuadas en Chile, en donde se evidencian aspectos de carácter físico, ambiente y factores psicosociales. Debido a esto, la evidencia de estudios identifica:

- Descripción de definiciones de concepto.

- Determinar referencia histórica sobre la temática.

- Estudios que avalen la prevalencia de factores que inciden en el área de estudio.

- Identificar metodología asociada.

- Reflexionar sobre la situación actual y afecciones futuras como una forma de prevención.

El alcance del estudio tiene por objetivo lograr una incorporación al control de salud del trabajador dedicado a la atención hospitalaria y un seguimiento propiamente tal, ya que se evidenciarán las posibles falencias y riesgos que puedan perjudicar su estado de salud. Con esto, se generará un plan de contingencia que identifique las variables que presenta el trabajador en relación a un análisis del sistema de turno (cuarto turno), afecciones físicas, factores de riesgo psicosociales, organizacionales y el ambiente en el proceso de atención en salud, que permitan llevar a cabo un mejor control de su labor.

Para realizar el análisis se llevó a cabo la revisión bibliográfica con acceso virtual al Sistema de Bibliotecas de la Universidad de Concepción (SIBUDEC) e investigaciones relacionadas a la temática de análisis.

Se utilizaron diferentes motores de búsqueda como revistas científicas Ergonomic, Cielo, entre otros. Y algunos artículos de revistas electrónicas relacionadas con el tema como Applied Ergonomics, Open Journal y Ergonomics.

Se encontraron más de 40 investigaciones asociadas a la temática, pero se privilegió la materia de carácter físico, mental, organizacional y diseño de jornada de trabajo con el propósito de identificar deficiencias propiamente tales del sistema de salud chileno.

\section{DESARROLLO DEL TEMA}

\section{Antecedentes históricos y algunas definiciones conceptuales}

El término ergonomía, en sus inicios, fue mencionado por el "naturalista Polaco Woitej Yastembowsky en 1857 en su estudio Ensayos de Ergonomía o Ciencias del Trabajo, basado en las leyes objetivas de la ciencia sobre la naturaleza, en la cual se proponían construir un modelo de la actividad laboral humana". Sin embargo, fue el "Psicólogo Británico K.F.H. Murrell quien definió el término en 1949, cuando un grupo de científicos se reunió en Inglaterra para formar la Sociedad de Investigaciones Ergonómicas", cuyo propósito en ese entonces era formar diversos profesionales que tuvieran interés en el mejoramiento del hombre en el trabajo y un avance en el estudio del "Comportamiento humano en el trabajo".

Murrell, en 1969, en su libro "Ergonomics" indica cuáles fueron sus ideales para propiciar el uso del vocablo ergonomía, para que este fuera traducido sin dificultad, por lo que analiza y realiza una descripción de la palabra Ergonomía, indicando su procedencia de dos palabras griegas: "Ergo (trabajo) y Nomos (leyes, reglas), cuyo significado corresponde a leyes o reglas del trabajo". Zander, en 1986, establece que "la ergonomía es el estudio del hombre en el trabajo, con el propósito de lograr un óptimo sistema hombre-tarea, en el cual pueda mantenerse un adecuado balance entre el trabajador y las condiciones laborales"1,2
Finalmente, "El Consejo Internacional Ergonomics Association (IEA), el cual reúne a todas las sociedades científicas a nivel mundial, establece desde el año 2000 lo siguiente: Ergonomía o factores humanos es la disciplina científica relacionada con la comprensión de las interacciones entre los seres humanos, los elementos de un sistema, la profesión que aplica la teoría, principios, datos, métodos de diseño para optimizar el bienestar humano y todo el desempeño del sistema",2,3

Cabe destacar que la incorporación de la ergonomía en el ámbito laboral ha propuesto mejoras al respecto tanto en el bienestar de los trabajadores como en la productividad en las empresas; sin embargo, es necesario que exista eficiencia y seguridad en los trabajos, debido a que es importante que el personal cuente con condiciones favorables para desarrollar sus actividades respectivas. Es por esto que, dentro de las áreas de trabajo en Chile, existe un organismo que compromete el bienestar de la población trabajadora: en el caso del personal de atención hospitalaria en Chile, la cantidad de trabajo se evidencia en niveles deficientes en cuanto a su desempeño y cantidad de horas laborales, debido al diseño de los sistemas de trabajo, carga mental, y existencia de alguna prevalencia a padecer trastornos músculo-esqueléticos relacionados con el trabajo.

Si bien es importante destacar que, en cuanto al sector de la salud, la ergonomía presenta análisis de casos estudiados e investigaciones respecto a los principales riesgos ocupacionales en relación con elementos de carácter físico, cognitivo, factores psicosociales, organizacionales y ambientales. Es por esto que existe una estrecha relación con posibles dolencias músculo-esqueléticas (relacionadas con el trabajo), el diseño del puesto y sistema de trabajo y, finalmente, la carga mental asociada a la actividad efectuada.

\section{Investigación sobre riesgos ergonómicos en la atención hospitalaria en Chile}

En Chile, la población que atiende centros hospitalarios presenta una caracterización en cuanto a sus capacidades, limitaciones y estado de salud ligado al desempeño y desarrollo de sus actividades de atención; en la Séptima Encuesta Laboral 2011 se identifica lo siguiente: "Factores de riesgo biomecánicos en relación con daño al sistema músculo-esquelético para actividades económicas agrupadas en servicios sociales y de la salud", en donde se establecen los siguientes datos:

\section{Tabla 1.}

\begin{tabular}{lr}
\hline Exposición & $\%$ \\
\hline Organizaciones con trabajadores expuestos a posturas incómodas & $18,6 \%$ \\
Cargas pesadas & $15,7 \%$ \\
Movimientos repetitivos & $14,2 \%$ \\
Organización del trabajo (exposición a turnos) en servicios sociales y salud & $23,7 \%$ \\
Ambiente fisico: & \\
Ruido & $10,7 \%$ \\
Altas temperaturas & $8,4 \%$ \\
Vibraciones mecánicas & $2,4 \%$ \\
Falta de iluminación & $0,6 \%$
\end{tabular}

Fuente: Dirección del Trabajo. Séptima Encuesta Laboral 2011.

Dentro de la encuesta laboral, no se encontraron registros de encuestas a nivel de riesgos por carga mental, riesgo psicosocial y demandas del trabajo en el sector de salud. Sin embargo, existe mayor investigación en "el área de la salud en Chile, sobre trastornos músculo-esqueléticos, carga física y turnos de trabajo. En la carga física, se han estudiado labores relacionadas con el manejo de 
pacientes, en demandas biomecánicas de las tareas así como la prevalencia de trastornos músculo-esqueléticos y factores de riesgos asociados" 4,5

Los trastornos músculo-esqueléticos hacen referencia a un conjunto de alteraciones de músculos, tendones, articulaciones, nervios y sistema vascular, de diferentes regiones del cuerpo, cuya mayor frecuencia se presenta en zona lumbar, cuello y extremidades superiores. La lesión puede ser originada por trauma acumulativo, la cual se desarrolla gradualmente en un periodo de tiempo, como resultado de demandas asociadas, entre otros factores de fuerza, repetitividad, sobrecarga postural y ausencia de períodos de recuperación. ${ }^{6}$

\section{Teoría multifactorial o de interacción multivariada en la generación de trastornos músculo-esqueléticos}

Al respecto, se realizó un estudio de antecedentes registrados de funcionarios de centros hospitalarios. ${ }^{4}$ Se clasificaron los potenciales factores de riesgo indentificados en categorias, que se relacionan con: los procedimientos de trabajo, equipamiento, la organización del trabajo, la infraestructura y características de las personas. En cada categoría, se pueden identificar factores de riesgo específicos. De este modo, para ordenar la gama de factores se empleó el diagrama causa-efecto de Ishikawa, el cual muestra una representación de antecedentes investigados a modo de establecer problemas y soluciones de forma más eficiente. ${ }^{6}$

A través de este análisis, se pueden evidenciar los antecedentes expuestos a riesgos y clasificarlos, con el propósito de desarrollar un mejor plan para disminuir riesgos presentes o encontrados para este caso, labores intrahospitalarios.

En cuanto a los turnos de trabajo en el sector chileno de la salud, "Numerosas investigaciones han demostrado que el trabajo en turnos y nocturno puede comprometer la salud, bienestar y rendimiento laboral".

En Chile, estudios realizados por la Dirección del trabajo indican que el 23,2\% de las empresas utiliza esta forma de organización del tiempo laboral. ${ }^{11}$ "En el sector de la salud chileno, el esquema tradicional aplicado desde al menos 50 años es el denominado "cuarto

Figura 1.

Diagrama causa-efecto de Ishikawa para TMEs.

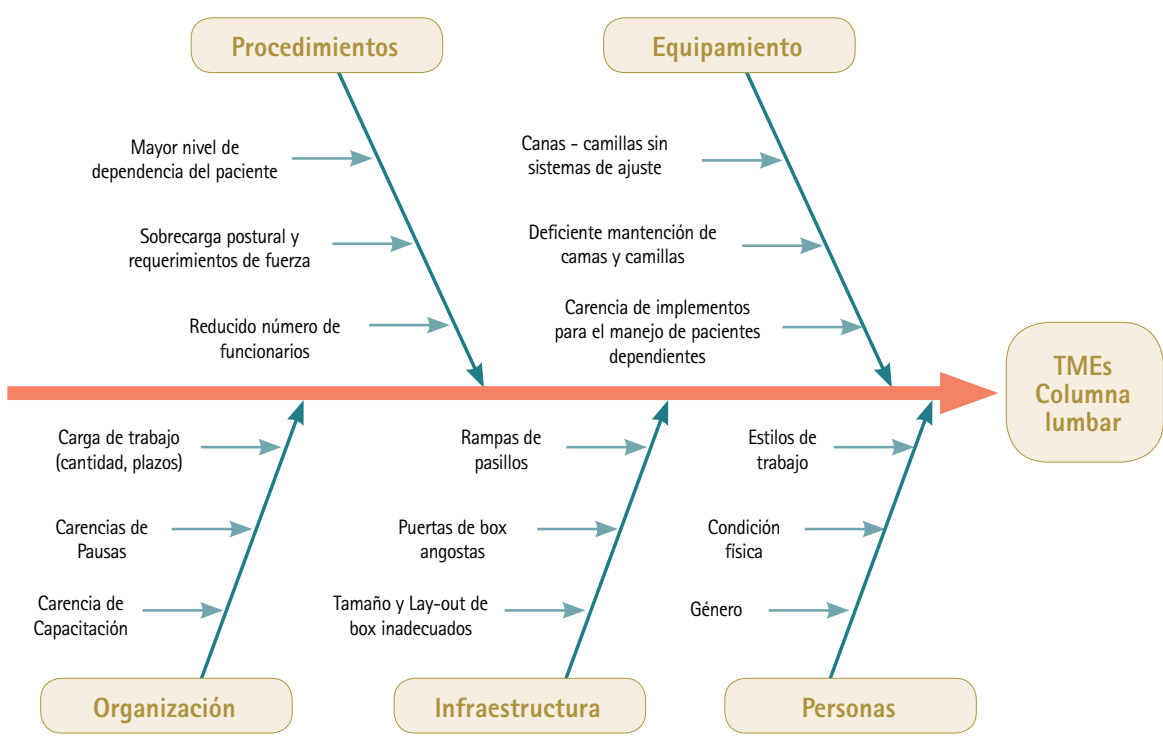

turno", el cual corresponde a un sistema que implica trabajar una jornada diurna de 12 horas, luego una jornada nocturna de la misma duración para después acceder a 2 días libres".12

En una investigación se realizó una comparación entre el sector público y privado, en donde "se presume que el sector público presentaría alteraciones significativamente superiores a las que presenta el personal que se desempeña en el sector privado". "Se encuestó una muestra de 44 trabajadores de un hospital privado y 43 trabajadores de un hospital público, ambos localizados en Santiago de Chile". ${ }^{13}$

La evaluación que se presenta de este sistema de turnos permite concluir que no se presenta riesgo significativo para las personas en los ámbitos de la salud, bienestar, vida social, adaptación y rendimiento. No existen diferencias significativas en la percepción de fatiga física, alteraciones sociales y presencia de trastornos del sueño entre el personal del sector privado y público. Las principales debilidades del sistema de cuarto turno son su alta irregularidad y sus principales fortalezas son el alto número de días libres por semana, moderado impacto sobre el rito sueño-vigilia y moderada desvisculación social y familiar. ${ }^{13}$

\section{RIESGOS PSICOSOCIALES}

Respecto al análisis sobre factores psicosociales en el lugar de trabajo, este se define por:

"Conjunto de condiciones relacionadas con la organización y contenido de las tareas, con los procedimientos y métodos de trabajo, así como con las relaciones entre los trabajadores y con sus superiores, cuya exposición prolongada en el tiempo aumenta la posibilidad de experimentar tensión psíquica la que, una vez acumulada residualmente, será un precursor de los trastornos o problemas de salud y afectará la productividad. Por tanto, están estrechamente ligados al ausentismo, motivación y desempeño". ${ }^{14}$

Una investigación realizada sobre "Indicadores de salud mental asociados a riesgo psicosocial laboral en un hospital público" demuestra en su análisis en primera instancia que "en el contexto laboral emergen los denominados riesgos psicosociales laborales, que aluden a un conjunto de características de la organización y de las condiciones de trabajo que afectan la salud de los trabajadores. Generando efectos en la salud cardiovascular, osteomuscular y en la salud mental".15 Además, en la investigación se establece lo siguiente:

Los trabajadores de salud están expuestos a altos niveles de estrés y riesgos psicosociales. El desequilibrio entre los esfuerzos invertidos y recompensas recibidas adquiere especial importancia en este contexto. El objetivo está dado por evaluar el nivel de riesgo psicosocial y su relación con la depresión, la angustia y el uso de drogas psicotrópicas entre los trabajadores de la salud. Los materiales y métodos corresponden a evaluar setecientos ochenta y dos trabajadores (602 mujeres y 180 hombres), los cuales respondieron cuestionarios autoadministrados para medir el riesgo psicosocial y salud mental. Los resultados son: el 25\% de 
los encuestados utilizan psicofármacos, el 34\% tenía un alto nivel de angustia y el 23\% tenía síntomas depresivos. También informaron de un nivel bajo de latitud decisional (48\%), demandas emocionales altas (47\%), apoyo social bajo (41\%) y un desequilibrio esfuerzorecompensa significativa (67\%). Las personas expuestas a la tensión laboral (alta demanda y baja latitud de decisión), iso-deformación (tensión laboral más bajo apoyo social), y el desequilibrio esfuerzorecompensa tenían el doble de probabilidades de sufrir síntomas de depresión y angustia elevada en comparación con sujetos no expuestos. Por ende, se concluye que existen altos niveles de estrés psicosocial entre los trabajadores de la salud. ${ }^{16}$

Si bien entre los factores psicosociales se hace presente el estrés como un estado generado por la actividad evaluada correspondiente a la atención hospitalaria, la cual requiere de una alta demanda de trabajo y resolver situaciones, junto con tomar decisiones complejas al momento de presentar instancias con una mayor demanda. Por otro lado, contar con un horario de jornada larga puede generar fatiga y distorsión del ciclo del sueño (ciclos circadianos).

\section{CONCLUSIONES}

La importancia de evaluar factores de riesgo ergonómico en la atención hospitalaria en Chile es una forma eficiente de mantener registros tanto físicos como mentales sobre el estado de salud de los trabajadores dedicados a esta labor, ya que se evidenciarán falencias y posteriormente un plan de mejoramiento y fortalecimiento del área. Por esto, es pertinente realizar una evaluación en forma anual en los trabajadores para llevar un seguimiento y control, así proponer cambios dentro del sistema de trabajo, contar con mayor personal y reestructurar el área de trabajo en el caso que sea necesario. Además, evidenciar riesgos de trastornos músculo-esqueléticos como una forma de prevención y mejorar las condiciones físicas del trabajador.

Luego de analizar el tema de estudio se pueden establecer los siguientes acuerdos y desacuerdos:

- Existe una preocupación tanto por las condiciones de trabajo y las deficiencias en la salud de los trabajadores dedicados a la atención hospitalaria en Chile, que se puede apreciar en la séptima encuesta laboral 2011, donde el factor repetitividad y trabajadores expuestos a posturas incómodas se encuentran sobre el 15\%, generando una preocupación por su salud y la calidad de atención por la aparición de antecedentes de riesgos.

- Se evidencian enfermedades asociadas por un desgaste físico y mental en el personal de salud, ya que a nivel físico se registran riesgos de trastornos músculo-esqueléticos a nivel de extremidad superior, las cuales se ven expuestas por movimientos, posturas inadecuadas y carga pesada. Por otro lado, riesgos mentales debido a la prevalencia existente en altos niveles de estrés psicosocial entre los trabajadores de la salud junto con el ambiente de exposición y sistema de turnos de trabajo, donde se aprecia un moderado impacto sobre el rito sueño-vigilia y moderada disvinculación social y familiar.

- Al llevar un control en la salud de los trabajadores, se evidenciarán riesgos y falencias que podrán ser mejoradas con un plan de carácter organizacional que proporcione un mejoramiento en el personal.

Cabe destacar el acuerdo del estudio realizado sobre la evidencia encontrada y las vías de mejoramiento para poder lograr un cambio en las condiciones de los trabajadores, una preocupación por la salud, evitar enfermedades crónicas por malos hábitos y falta de preocupación.
1. Jaureguiberry ME. Ergonomía [en línea]. Buenos Aires: UNICEN-Prov. B. Aires; [f.d.] [consultado abr 2015]. 9 p. Disponible en: http://www.fio.unicen.edu.ar/ usuario/segumar/Laura/material/ERGONOMIA.pdf

2. Apud E, Meyer F. La importancia de la ergonomía para los profesionales de la salud. Cienc enferm. 2003; 9(1):15-20.

3. International Ergonomics Association. Definition and Domains of Ergonomics [on line]. Zurich: IEA; 2016 [cited abr 2015]. Available from http://www.iea.cc/whats/

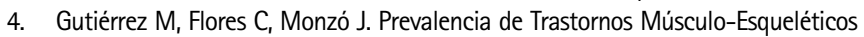
en Funcionarios de Centros Hospitalarios que Realizan Manejo de Pacientes y Caracterización de Potenciales Factores de Riesgo. Cienc Trab. 2010; 12(38):447453

5. Dirección del Trabajo. Séptima Encuesta Laboral 2011. Informe de resultados [en linea]. Santiago de Chile: Dirección del Trabajo; 2011 [citado abr 2015]. Disponible en: http://www.dt.gob.cl/documentacion/1612/articles-101347_ recurso_1.pdf.

6. Gutiérrez M, Monzó J, Lama O, Felmer A, Cruzat M, Bustos G. Ergonomics applied to risk management of musculoskeletal disorders in Clinical Laboratory of Public Hospital [en línea]. Concepción: Universidad de Concepción; [2012] [citado abr 2015]. Disponible en: http://ergonomics.uq.edu.au/iea/proceedings/ Index_files/papers/276.pdf

7. Costa G. The impact of shift and night work on health. Appl Ergon. 1996; 27(1):9-16.

8. Duchon J, Smith T. Extended workdays and safety. Ergonomics. 1993;11:37-49.

9. Fischer FM, Paraguay Al, De Castro A, Moreno C, Berwerth A, Riviello C, et al. Working conditions, work organization and consequences for health of Brazilian petrochemical workers. Int J Ind Ergon. 1998; 21:209-219.

10. Monk $T H$, Folkard $S$, Wedderburn, A. Maintaining safety and high performance on shiftwork. Appl Ergon. 1996; 27(1):17-23.
11. Espinosa M, Damianovic N. Encuesta Laboral ENCLA. Informe de Resultados. Santiago: Dirección del Trabajo de Chile; 2000.

12. Maulen J. Falencias de enfermeras [en linea]. Santiago: Colegio de Enfermeras de Chile; 1999 [citado abr 2015]. Disponible en: http://www.colegiodeenfermeras.cl/eleccion.html \#investigaciones.

13. Córdova V, Hevia JC, Figueroa A. Trabajo en turnos en el sector de la salud chileno: una comparación entre el sector público y privado. Cienc Trab. 2006; 8(21):147-150.

14. Moreno B, Báez C. Factores y riesgos psicosociales, formas, consecuencias, medidas y buenas prácticas [en línea]. Madrid: INSHT; 2010 [citado abr 2015]. Disponible en: http://www.insht.es/InshtWeb/Contenidos/Documentacion/ PUBLICACIONES\%2OPROFESIONALES/factores\%20riesgos\%20psico.pdf.

15. Organización Internacional del Trabajo (OIT)-Organización Mundial de la Salud (OMS). Factores psicosociales en el trabajo: naturaleza, incidencia y prevención. Ginebra: OIT-OMS; 1984.

16. Ansoleaga E. Indicadores de salud mental asociados a riesgo psicosocial laboral en un hospital público. Rev méd Chile [en línea]. 2015 [citado abr 2015];143(1), 47-55. Disponible en: http://www.scielo.cl/scielo.php?script= sci_arttext\&pid= S0034-98872015000100006\&Ing=es\&tlng=es. 10.4067/ S0034-98872015000100006.

\section{Otras referencias complementarias:}

Gutiérrez M. Ergonomía e investigación en el sector salud. Cienc enferm [en línea]. 2014 [citado abr 2015]; 20(3):7-10. Disponible en: http://www.scielo.cl/scielo. php?script=sci_arttext\&pid=S0717-95532014000300001\&lng=es\&tlng=es. 10.4067/S0717-95532014000300001.

Putz-Anderson V. Cumulative trauma disorders: A manual for musculoskeletal diseases of the upper limbs. London: Taylor \& Francis; 1988. 